\title{
Comparative study of mixed product and quaternion product
}

\author{
Md. Shah Alam \\ Department of Physics. Shahjalal University of Science and Technology, \\ Sylhet, Bangladesh \\ E-mail

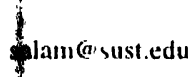

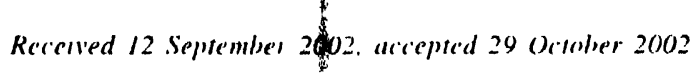

\begin{abstract}
Mixed number is the sum of a scalar and a vector The quatemon can also be written as the sum of a scalar and a vector but the product of imixed numbers and the product of qualernions are different. Here, we studied the mixed product which is derived from the product of mixed numbers and the quaternion product which is derived froin the product of quaternions li was observed that mixed product is more consistent with Physies than that of quaternion product.
\end{abstract}

kiswords . Mixed number, mixed product, quaternion product

PACS No. $\cdot 0290+p$

\section{Introduction}

\subsection{Mixed product :}

Mixed number [1] $\alpha$ is the sum of a scalar $x$ and $a$ vector $\boldsymbol{A}$ :

$$
\text { t.e. } \alpha=x+A \text {. }
$$

The product of two mixed numbers is defined as [1]

$$
\alpha \beta=(x+A)(y+B)=x y+A \cdot B+x B+y A+i A \times B .
$$

Taking $x=y=0$, we get from eq. (1)

$$
A \otimes B=A . B+i A \times B .
$$

This product is called mixed product $[2\}$ and the symbol $\otimes$ is chosen for it.

\subsection{Quaternion product :}

The quaternion can also be written as the sum of scalar and a vector [3]

$$
\text { i.e. } \underline{A}=a+\boldsymbol{A} \text {. }
$$

The multiplication of any two quaternions $A=a+A$ and $B=b+B$ is given by [3-5]

$$
\text { Corresponding Author }
$$

$$
\underline{A B}=(a+A)(b+B)=a b-A \cdot B+a B+b A+A \times B .
$$

Taking $a=b=0$, we get from eq. (3)

$$
\underline{A B}=-\boldsymbol{A} \cdot \boldsymbol{B}+\boldsymbol{A} \times \boldsymbol{B} .
$$

This product we call quaternion product.

\section{Consistency of mixed product and quaternion product with} physics

2.1 Consistency with Pauli matrix algebra:

It can be shown that $|6|$

$$
(\sigma \cdot A)(\sigma \cdot B)=\boldsymbol{A} \cdot \boldsymbol{B}+i \sigma \cdot(\boldsymbol{A} \times \boldsymbol{B}),
$$

where $A$ and $B$ are two vectors and $\sigma$ is the Pauli matrix.

From eqs. (2) and (5), we can say that the mixed product is directly consistent with Pauli matrix algebra. From eqs. (4) and (5), we can also say that the quaternion product is not directly consistent with Pauli matrix algebra.

\subsection{Consistency with Dirac equation :}

Dirac equation $(E-\alpha . P-\beta m) \psi=0$ can be operated by the Dirac operator $(t-\alpha . V-\beta n)$, then we get

$$
(t-\alpha . V-\beta m)\{(E-\alpha . P-\beta m) \psi\}=0 \text {. }
$$


For mass-less particles i.e. for $m=n=0$, we get [7]

$$
\begin{aligned}
& (t-\alpha . V)(E-\alpha . P) \psi=\{t E+V . P+i \sigma .(V \times P)\} \psi_{1} \\
& +\{-(t \sigma . P+E \sigma . V)\} \psi_{2}=0,
\end{aligned}
$$

where $\psi$ is the wave function and $\psi_{1}$ and $\psi_{2}$ are the components of $\psi$.

Putting $t=0$ and $E=0$ in eq. (7), we get

$$
(\alpha . V)(\alpha . P) \psi=\{\boldsymbol{V} . \boldsymbol{P}+i \sigma \cdot(\boldsymbol{V} \times \boldsymbol{P})\} \psi_{1}=0 .
$$

From eqs. (2) and ( 8 ), it is clear that Mixed product is consistent with Dirac cquation.

From cqs. (4) and ( 8 ), it is also clear that quaternion product is not consistent with Dirac equation.

\section{Applications of these products in dealing with differential operators}

In region of space where there is no charge or current, Maxwell's equation can be written as

(i) $\nabla . E=0, \quad$ (ii) $\nabla \times E=-(\partial B) /(\partial t)$,

(iii) $\nabla . B=0, \quad$ (iv) $\nabla \times B=\mu_{0} \varepsilon_{0}(\partial E) /(\partial t)$.

From these equations, it can be written as [8]

$$
\begin{aligned}
& \nabla^{2} E=\mu_{0} \varepsilon_{0}\left(\partial^{2} E\right) /\left(\partial t^{2}\right), \\
& \nabla^{2} B=\mu_{0} \varepsilon_{0}\left(\partial^{2} B\right) /\left(\partial t^{2}\right) .
\end{aligned}
$$

Using eqs. (2) and (9), we can write

$$
\begin{aligned}
& \nabla \otimes E=\nabla \cdot E+i \nabla \times E \\
& =0+-i(\partial B) /\left(\partial_{t}\right) .
\end{aligned}
$$

or

$$
\nabla \otimes E=-i(\partial B) /(\partial t)
$$

or

$$
\begin{aligned}
\nabla \otimes(\nabla & \otimes E)=\nabla \otimes\{-i(\partial B) /(\partial t)\} \\
& =-i(\partial / \partial t)\{\nabla \otimes B\} \\
& =-i(\partial / \partial t)\{\nabla \cdot B+i \nabla \times B\} \\
& =-i(\partial / \partial t)\left\{0+i \mu_{0} \varepsilon_{0}(\partial E) /(\partial t)\right\}
\end{aligned}
$$

or $\quad \nabla \otimes(\nabla \otimes E)=\mu_{0} \varepsilon_{0}\left(\partial^{2} E\right) /\left(\partial t^{2}\right)$.
It can be shown that $\nabla \otimes(\nabla \otimes E)=\nabla^{2} E$.

From eqs. (12) and (13), we can write

$$
\nabla^{2} E=\mu_{0} \varepsilon_{0}\left(\partial^{2} E\right) /\left(\partial t^{2}\right)
$$

which is exactly same as shown in eq. (10).

Similarly using mixed product, it can also be shown that

$$
\nabla^{2} B=\mu_{0} \varepsilon_{0}\left(\partial^{2} B\right) /\left(\partial t^{2}\right) .
$$

Therefore, mixed product can be used successfully in dealing with differential operators. Using the definition of quaternion product (eq. 4), it can be shown that quaternion product can not be used in dealing with differential operators.

\section{Elementary properties of these products}

\subsection{Elementary properties of mixed product:}

(i) Mixed product of two perpendicular vectors is equal in the imaginary of the vector product of the vectors

(ii) Mixed product of two parallel vectors is simply the scalar product of the vectors.

(iii) It satisfies the distribution law of multiplication.

(iv) It is associative.

4.2 Elementary properties of quaternion product :

(i) Quaternion product of two perpendicular vectors is simply the vector product of the vectors.

(ii) Quaternion product of two parallel vectors is negativi of the scalar product of the vectors.

(iii) It satisfies the distribution law of multiplication.

(iv) It is non-associative.

\section{Conclusion}

A comparison of Quaternion and mixed products is given in Table 1. From the table we may conclude that mixed product is more consistent with different laws of Physics than that Quaternion product. From the table we may conclude that mixed product is more consistent with different laws of Physics that the Quaternion product.

\section{Acknowledgments}

I am grateful to Mushfiq Ahmad, Department of Physics. University of Rajshahi, Rajshahi, Bangladesh and Prof. Habibul Ahsan, Department of Physics, Shahjalal University of Science and Technology, Sylhet, Bangladesh and Prof. Anwarul Haque Sharif, Department of Mathematics, Jahangir Nagar University. Dhaka, Bangladesh for their help and advice. 
Table 1. Comparison of quaternon product and mixed product

Quaternion product

I. Mathematical expression

2. Consistency with Pauli matrix algebra

3. Consistency with Dirac equation

4. In dealing with differential operators

5. Elementary properties

$$
\underline{A B}=A . B-A \times B
$$
with Pauli matrix algebra equation : ,
It is not directly consistent

It is not consistent with Dirac

It can not he used in dealing with differential operators

(i) Quaterhion product of Iwo perpendicular vector is simply the vectoryproduct of the vectors.

(ii) Quaternion product of two parallel vectors is negative of the scalar product of the vectors.

(iii) It satisfies the distribution law of multiplication.

(iv) It is non-associative.
Mixed product

$A \otimes B=A . B+i A \times B$

It is derectly consistent with Pauli matrix algebra

It is consistent with Dirac equation

It can be used successfully in dealing with differential operators

(i) Mixed product of (wo) perpendicular vectors is equal to the imagmatry of the vector product of the veclors

(ii) Mixed producl of Iwo parallel vectors is simply the scialar product of the vectors.

(iii) It saltefies the distribution lat of muluplicatıon.

(Iv) It is associative

\section{Rolerences}

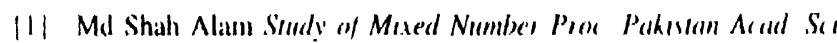
$37119(2000(1)$

121 Md Shah Alam Mered Product of Vertors J Theroleters Vol 3 (20)1)

13 A Kyrala Theoneencal Phisess (Philadelphia \& London W B Saunders Company) (Toppan Company L.muted Tokyo. Japan) (1967)
14] hup //mathwold wollian com/Qualcrmon hrml

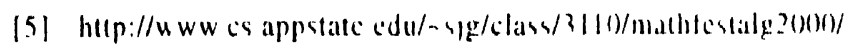
quaternsons $/ \mathrm{html}$

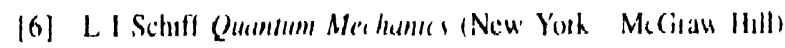

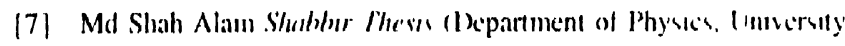
of Rajshahr. Rapshahı. Bangladesh) (1994)

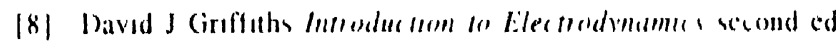
(New Delhu Prentice Hall of Indra) (199.7) 